\title{
Modified Adaptive Fusion Scheme for Kalman Filter Based on the Hypothesis Test
}

\author{
Chen Jiang $\mathbb{D}$, Wenkai Liu $\mathbb{D}$, Hui Li $\mathbb{D}$, and Haijun Xu $\mathbb{1}$ \\ College of Surveying and Geo-Informatics, North China University of Water Resources and Electric Power, \\ Zhengzhou 450045, China \\ Correspondence should be addressed to Haijun Xu; xuhaijun@ncwu.edu.cn
}

Received 4 June 2021; Accepted 5 January 2022; Published 29 January 2022

Academic Editor: Qiu-Zhao Zhang

Copyright (c) 2022 Chen Jiang et al. This is an open access article distributed under the Creative Commons Attribution License, which permits unrestricted use, distribution, and reproduction in any medium, provided the original work is properly cited.

In the literature, the fading factor was constructed to overcome the shortage of model uncertainties in the Kalman filter. However, the a priori covariance matrix might be inflated abnormally by the fading factor once the measurement is unreliable. Thus, the fading factor may become invalid, and this problem is rarely discussed and tested. In this paper, squares of the Mahalanobis distance are introduced as the judging index, and the fading factor or the covariance inflation factor is adopted conditionally according to the hypothesis testing result. Therefore, an adaptive filtering scheme based on the Mahalanobis distance is put forward for the systems with model uncertainties. The proposed algorithm is implemented with the actual data collected by the integration of the global navigation satellite system (GNSS) and the inertial navigation system and INS (inertial navigation system) integrated systems (INS). For the systems with model uncertainties, experimental results demonstrate that the influences of both the outlying measurements and model errors are controlled effectively with the proposed scheme.

\section{Introduction}

As the linear estimator of the mean and covariance, the Kalman filter has become the classic fusion algorithm in many fields $[1,2]$, and it is implemented as the basic fusion method in the data processing with multiple sensors [3, 4]. It has been proved that the Kalman filter is optimal only when the assumptions of Gaussian-distributed process or measurement noise hold [5]. Unfortunately, the Kalman filter is susceptible to outlying measurements, and it performs inadequately with the model errors and uncertain statistical information. Aiming at the outlying measurements, many robust Kalman filtering algorithms were proposed. Outlier detection for all measurements is the most straightforward strategy, and the measurements with relatively large residuals will be rejected [6]. Nevertheless, all measurements should be tested, and this strategy may be inefficient and complex. The filters based on the median may be highly robust; however, many measurements are ignored, and the low efficiency limits its practical applications [7]. The Ho filter was put forward aiming at the uncertain noises of mea- surement with the minimized estimation error for all possible disturbances [5]. However, the performance would be degraded significantly by the outliers [8]. As the generalized Kalman filter, the Bayesian estimator was derived robustly based on the M-estimation [9]. DIA (detection, identification, and adaptation) methods were developed to resist the influences of outliers, but the identification is valid only when the measurement is reliable [10]. Influences of the model errors can be weakened with many types of adaptive filters [11-13]. In terms of adaptive filter, the MMAE (multiple-model-based adaptive estimation) and IAE (innovation-based adaptive estimation) [14] are two basic strategies. For the data fusion of GNSS/INS integrated systems, the IAE strategy performs better than the MMAE strategy [11]. Besides, another type of adaptive-robust filter where both the adaptation and robustness were considered simultaneously was developed using the adaptive factors and the M-estimation, including four adaptive factors and four detective statistics [15]. Based on this adaptive-robust filter, the influences on the adaptive factor from the outlying measurements were discussed [16], and an alternative strategy 
was constructed to control the influences of both model uncertainties and outliers. However, the covariance of the measurement noises remains constant at the normal epochs, and the relatively smaller outliers are more likely to be neglected which should be improved further.

In theory, the fading filter is another kind of adaptive filter, and it can control the influences of the dynamic model errors[17]. The most important problem for the fading filter is to construct a suitable factor $S_{k}$. Then, the fading matrix was proposed to adjust the covariance matrix $P_{k / k-1}$ in different data channels $[18,19]$, and the fading matrix has been adopted in the data processing of GNSS/INS integrated systems [20]. The main superiority to the conventional Kalman filter lies on inflating the covariance matrix of the a priori state, namely, $P_{k / k-1}$, and this indicates that the state estimation $\widehat{X}_{k / k}$ relies more on the current observation information. However, both the single factor and the fading matrix are constructed based on the residual vector. Therefore, the fading filter performs well only when the measurement is reliable, and the filter divergence may happen in the presence of outlying measurements, but this problem is seldom discussed. Consequently, aiming at the influences of outlying measurements, the robustness of the conventional fading filter should be improved further.

In this paper, a modified adaptive filtering scheme using the fading factors is proposed for the system with model uncertainty, and the Mahalanobis distance from the measurement to its prediction is applied to construct the judging index for the hypothesis test. Whether the fading filter or the robust estimation method is performed at each epoch depends on the result of hypothesis test. The proposed algorithm is tested using the actual data obtained through the self-developed GNSS/INS integrated systems in actual environment. Both the conventional fading filter and the proposed algorithm are implemented in the testing section of this paper. Results demonstrate that the proposed scheme is superior among all the other tested algorithms of this paper with or without the outlying measurements, and the filter divergence is restrained with the proposed algorithm.

Remaining of this paper is arranged as follows. The related theory of the fading filter is introduced in Section 2. In Section 3, a modified adaptive data fusion scheme is constructed based on the hypothesis test and the conventional fading filter. In Section 4, the dynamic model and measurement model for the GNSS/INS integrated systems are provided; then, a flowchart of the proposed algorithm is demonstrated. In Section 5, experiments with actual data are implemented, and performance the comparative algorithms together with the proposed scheme is tested. Section 6 provides the conclusions of this paper.

\section{The Fading Filter}

2.1. Basic Models of the Fading Filter. Kalman filter performs well when the assumptions of Gaussian distribution hold. However, the filter divergence may happen, resulting from big model errors. Aiming at this problem, the fading filter was proposed to limit the "memory length" of the Kalman filter [21]. Assume that $x_{k}$ and $x_{k-1}$ are the state vector at epoch $k$ and $k-1$, respectively, $\Phi_{k / k-1}$ denotes the state transition matrix, and $w_{k}$ is the state noise matrix; then, the dynamic equation is obtained

$$
x_{k}=\Phi_{k / k-1} x_{k-1}+w_{k},
$$

and the a priori state is given by

$$
x_{k / k-1}=\Phi_{k / k-1} x_{k-1} .
$$

Assume that $H_{k}$ and $z_{k}$ are the measurement matrix and measurement vector, respectively, $v_{k}$ is the measurement noise, and $S_{k}$ is the fading factor (in general, $S_{k} \geq 1$ ). Then, the optimality criterion is defined by [21]:

$$
\begin{aligned}
& V_{k}^{T} R_{k}^{-1} V_{k}+\frac{1}{S_{k}}\left(x_{k / k}-x_{k / k-1}\right)^{T} P_{k / k-1}^{-1}\left(x_{k / k}-x_{k / k-1}\right) \\
& \quad+\left(x_{k / k}-x_{k / k-1}\right)^{T} Q_{k}^{-1}\left(x_{k / k}-x_{k / k-1}\right)=\min .
\end{aligned}
$$

Then, the iterative solution is listed below:

$$
\begin{aligned}
x_{k / k} & =x_{k / k-1}+\bar{K}_{k}\left(z_{k}-H_{k} x_{k / k-1}\right), \\
\bar{K}_{k} & =\bar{P}_{k / k-1} H_{k}^{T}\left(H_{k} \bar{P}_{k / k-1} H_{k}^{T}+R_{k}\right)^{-1}, \\
\bar{P}_{k / k-1} & =S_{k} \Phi_{k / k-1} P_{k-1} \Phi_{k / k-1}^{T}+Q_{k},
\end{aligned}
$$

where $\bar{K}_{k}$ denotes the equivalent gain matrix, $\bar{P}_{k / k-1}$ denotes the equivalent covariance matrix of $x_{k / k-1}, P_{k-1}$ denotes the covariance matrix of $x_{k-1 / k-1}$, and $R_{k}$ and $Q_{k}$ are the covariance matrices of measurement and state noises, respectively.

In the fading filter, the covariance matrix of $x_{k / k-1}$ is inflated for $S_{k}$ times when compared with that of the conventional Kalman filter, and this indicates that the current measurement information is treated with bigger weight [21]. Consequently, the model errors brought from the previous state are weakened with the fading factor.

2.2. Construction of the Fading Factor or Matrix. In the fading filter, the main work concentrates on the construction of the reasonable factor. In theory, the factor $S_{k}$ should be inflated if errors of $\hat{x}_{k-1}$ is abnormal. A fading filter with the optimal fading factor was developed, and the simplified and applicable fading factor is given by [22]

$$
S_{k}=\max \left\{1, \frac{\operatorname{tr}\left(N_{k}\right)}{\operatorname{tr}\left(M_{k}\right)}\right\} \text {, }
$$

and $N_{k}=P_{V_{k}}-H_{k} Q_{k} H_{k}^{T}-R_{k}, M_{k}=H_{k} \Phi_{k / k-1} P_{k-1} \Phi_{k / k-1}^{T} H_{k}^{T}$, $P_{V_{k}}=E\left(V_{k} V_{k}^{T}\right)$, and $V_{k}=H_{k} x_{k / k-1}-z_{k}$, where $\operatorname{tr}(\cdot)$ means the trace of a matrix, $V_{k}$ is the residual vector, $P_{V_{k}}$ is the covariance matrix of $V_{k}$, and $\widehat{P}_{V_{k}}=(1 / k) \sum_{i=1}^{k} V_{i} V_{i}^{T}$.

Apart from the fading filter with a single factor, the fading matrix for the fading filter was developed [18]. Different from the single factor, the fading filter with the fading matrix is adjusted in multiple data channels according to the 
observabilities of the state vector elements. The fading matrix $S_{k}{ }^{\prime}$ is derived by

$$
\begin{aligned}
S_{k}{ }^{\prime} & =\operatorname{diag}\left(s_{1}, s_{2}, \cdots, s_{i} \cdots, s_{t}, 1, \cdots, 1, \cdots, 1\right), \\
s_{i} & =\max \left(1, \sqrt{\frac{\left[v_{i}(k)\right]^{2}}{\lambda_{i}^{2} j_{i i}(k) \varepsilon_{i}}-\frac{b_{i i}(k)}{j_{i i}(k)}}\right),(i=1,2, \cdots, t),
\end{aligned}
$$

where $t$ is the dimension of measurement equation, $v_{i}$ is the $i$ th element of $V_{k}, \lambda_{i}$ is the $i$ th observable element of $H_{k}, j_{i i}$ is the $i$ th diagonal element of $J_{k}$ and $J_{k}=\Phi_{k} P_{k-1} \Phi_{k}^{\mathrm{T}}, b_{i i}$ is the $i$ th diagonal element of $B_{k}, B_{k}=H_{k} Q_{k-1} H_{k}^{T}+R_{k}$, and $\varepsilon_{i}$ is the threshold determined by the Chi-square distribution under the predetermined significance level. In Equation (9), $s_{1}, s_{2}$, $\cdots, s_{t}$ are estimated adaptively, and the other elements in $S_{k}{ }^{\prime}$ should be 1 .

\section{Modified Adaptive Data Fusion Scheme}

As depicted in Section 2, the current measurement information is treated with bigger weight in the fading filter, and the current measurement information must be reliable to achieve an ideal filtering performance. In the fading filter however, the circumstance, when the current measurement information is unreliable, is rarely discussed and tested with actual data. In fact, both a single fading factor and the fading matrix in the fading filter are constructed by the predicted residual vector obtained from the measurements. Therefore, the performance of the fading factor would be affected easily by unreliable measurements, and a mistaken adjustment towards the a priori covariance matrix may result in inferior performance or even the filter divergence. Thus, the fading factor or matrix should be implemented in a timely and more reasonable way, and the strategy on processing the unreliable measurements should be discussed further.

With the Gaussian distribution process and measurement noise, the discrete linear stochastic state space model is given by [5]

$$
\left\{\begin{array}{l}
x_{k}=\Phi_{k / k-1} x_{k-1}+w_{k}, \\
z_{k}=H_{k} x_{k}+v_{k} .
\end{array}\right.
$$

An optimal solution is obtained if the assumptions of Gaussian distribution process and measurement noise hold with the mean $\bar{z}_{k}$ and covariance $P_{\bar{z}_{k}}$. Probability density function $\rho\left(z_{k}\right)$ of the $m$-dimensional measurement is [1]

$$
\begin{aligned}
\rho\left(z_{k}\right)= & N\left(z_{k} ; \bar{z}_{k}, P_{\bar{z}_{k}}\right) \\
& =\frac{\exp \left(-(1 / 2)\left(z_{k}-\bar{z}_{k}\right)^{T}\left(P_{\bar{z}_{k}}\right)^{-1}\left(z_{k}-\bar{z}_{k}\right)\right)}{\sqrt{(2 \pi)^{m}\left|P_{\bar{z}_{k}}\right|}} .
\end{aligned}
$$

Nevertheless, $\rho\left(z_{k}\right)$ would no longer hold once the outlying measurements exist or the measurement noises disobey the Gaussian distribution. Therefore, if $\rho\left(z_{k}\right)$ does not hold, and this in turn indicates that an outlier exists at some epoch or the Gaussian distribution of measurement noise is contaminated. Accordingly, the hypothesis test is performed with the null hypothesis that the measurement corresponds to the assumptions. In fact, the square of the Mahalanobis distance $M_{k}^{2}$ from the measurement $z_{k}$ to its mean $\bar{z}_{k}$, namely, $M_{k}^{2}=\left(z_{k}-\bar{z}_{k}\right)^{T}\left(P_{\bar{z}_{k}}\right)^{-1}\left(z_{k}-\bar{z}_{k}\right)$, is applied as the test statistic [23], and this test statistic has been adopted as to construct the robust Kalman filter [1]. According to the inertial assumptions, the test statistic should obey the Chisquare distinction with the degree of freedom $m$. Then, the $\alpha$-quantile $\chi_{\alpha}$ is obtained with the provided significance level $\alpha$. Obviously, the null hypothesis should be accepted if $M_{k}^{2}$ is less than $\chi_{\alpha}$; under this circumstance, no outliers exist, and the key problem is to weaken the influences of the model errors. Otherwise, the null hypothesis should be rejected, and the outliers should be processed carefully.

In order to weaken the influences of unreliable measurements, $R_{k}$ is estimated and updated in an adaptive way. In general, components in the vector of the a priori state are correlated; the covariance matrix of the measurement noise is updated with the covariance inflation factor $\lambda_{i j}$, namely,

$$
\begin{aligned}
& \lambda_{i j}=\sqrt{\lambda_{i i} \cdot \lambda_{j j},} \\
& \lambda_{i i}= \begin{cases}1 & \left|\bar{V}_{\bar{x}_{k_{i}}}\right| \leq c \\
\frac{\left|\bar{V}_{\bar{x}_{k_{i}}}\right|}{c} & \left|\bar{V}_{\bar{x}_{k_{i}}}\right|>c\end{cases}
\end{aligned}
$$

where $\left|\bar{V}_{\bar{x}_{k_{i}}}\right|$ denotes the standardized predicted residual and $c$ is fixed within $1.0 \sim 1.5$. Consequently, $R_{k}$ is rewritten as

$$
\bar{R}_{k}=\lambda_{i j} R_{k}
$$

In practice, the complex observation environment determines that $R_{k}$ is usually not a constant matrix, and the filter may perform better with adaptively updated $R_{k}$. Therefore, at the epochs with normal measurements, $R_{k}$ is updated adaptively in a sequential way based on the Sage-Husa filter [24]; in this paper and the influences of the outlying measurements will be weakened further, namely,

$$
R_{k}=\left(1-d_{k-1}\right) R_{k-1}+d_{k-1}\left(V_{k} V_{k}^{T}+H_{k} P_{k / k-1} H_{k}^{T}\right),
$$

where $d_{k}=(1-b) /\left(1-b^{k}\right)$ and $b$ is the forgetting factor.

With the hypothesis test based on the Mahalanobis distance, the outlying measurement is identified. Meanwhile, the covariances of the outlying measurement noises and the normal measurements noises will be updated through the Equations (14) and (15), respectively. Both the judging index $M_{k}^{2}$ and $R_{k}$ are computed using the current measurements, and this indicates a strong sensitivity towards the abnormal measurements and a relatively light computational burden. Accordingly, a modified adaptive data fusion scheme is constructed. In this proposed scheme, the hypothesis test based on the Mahalanobis distance is implemented 
at every epoch. For each epoch, if the null hypothesis is accepted, both the fading filter and the sequential updating of $R_{k}$ are implemented. Otherwise, only the equivalent covariance matrix $\bar{R}_{k}$ is performed, and this strategy is conducted adaptively. Therefore, a modified scheme is developed concerning the circumstance when the current measurement information is unreliable.

\section{Models for GNSS/INS Integrated Systems}

The GNSS/INS integrated systems have become a well applied technique in the field of dynamic navigation and positioning $[25,26]$. GNSS and INS are integrated mainly in three types, and the loosely coupled type is applied in this paper. For the GNSS/INS and various other integrated systems, the nonlinear filter should be implemented aiming at the nonlinear problem. The cubature Kalman filter (CKF) was proposed to address the high-dimensional state estimation [27]. With a fifteen-dimension state vector $\widehat{X}$, including the position error $\Delta R^{e}$, the velocity error $\Delta V^{e}$, the attitude error $\varphi^{e}$ of the carrier and the bias of the gyroscope $\nabla^{b}$, and the accelerometer $\varepsilon^{b}$, the CKF is adopted in this paper, where $e$ means the earth frame and $b$ means the body frame. Elements of $\widehat{X}$ are listed below.

$$
\widehat{X}=\left[\begin{array}{lllll}
\Delta R^{e} & \Delta V^{e} & \varphi^{e} & \nabla^{b} & \varepsilon^{b}
\end{array}\right]^{T},
$$

For the nonlinear discrete system

$$
\left\{\begin{array}{l}
x_{k}=f\left(x_{k-1}\right)+w_{k-1}, \\
z_{k}=h\left(x_{k}\right)+v_{k},
\end{array}\right.
$$

where $f(\cdot)$ and $h(\cdot)$ are the nonlinear functions. In the CKF, the time updating process is derived by $[27,28]$ :

$$
\begin{aligned}
X_{i / k-1} & =s_{k-1 / k-1} \xi+x_{k-1 / k-1}, \\
P_{k-1 / k-1} & =s_{k-1 / k-1} s_{k-1 / k-1}^{T}, \\
X_{i, k-1}^{*} & =f\left(X_{i, k-1}, w_{k}\right), \\
x_{k / k-1} & =\frac{1}{m} \sum_{i=1}^{m} w_{i} X_{i, k / k-1}^{*}, \\
P_{k / k-1} & =\frac{1}{m} \sum_{i=1}^{m} w_{i} X_{i, k / k-1}^{*} X_{i, k / k-1}^{* T}-x_{k / k-1} x_{k / k-1}^{T}+Q_{k} .
\end{aligned}
$$

And the measurement updating process is derived by

$$
\begin{aligned}
x_{k / k} & =x_{k / k-1}+K_{k}\left(z_{k}-z_{k / k-1}\right), \\
K_{k} & =P_{x z, k / k-1} P_{z z, k / k-1}^{-1}, \\
P_{k / k} & =P_{k / k-1}-K_{k} P_{z z, k / k-1} K_{k}^{T},
\end{aligned}
$$

$$
\left\{\begin{array}{l}
X_{k / k-1}=s_{k / k-1} \xi+x_{k / k-1}, \\
P_{k / k-1}=s_{k / k-1} s_{k / k-1}^{T}, \\
Z_{i, k / k-1}=h\left(X_{i, k / k-1}\right), \\
z_{k / k-1}=\frac{1}{m} \sum_{i=1}^{m} w_{i} Z_{i, k / k-1}, \\
P_{x z, k / k-1}=\frac{1}{m} \sum_{i=1}^{m} w_{i} X_{i, k / k-1} Z_{i, k / k-1}^{T}-x_{k / k-1} z_{k / k-1}^{T}, \\
P_{z z, k / k-1}=\frac{1}{m} \sum_{i=1}^{m} w_{i} Z_{i, k / k-1} Z_{i, k / k-1}^{T}-z_{k / k-1} z_{k / k-1}^{T}+R_{k}
\end{array}\right.
$$

where $X$ denotes the $m$ cubature points generated from states and $m=2 n, n$ denotes dimension of the state vector, $s_{k / k-1}$ denotes the square root of $P_{k / k-1}, \xi=\sqrt{(m / 2)}[1]_{i}$, and $X_{k / k-1}^{*}$ and $Z_{k / k-1}$ denote the propagated cubature points from states and measurements, respectively. Differences of the carrier position and velocity between GNSS and INS, namely, $\Delta r=r_{\mathrm{INS}}-r_{\mathrm{GNSS}}$ and $\Delta v=v_{\mathrm{INS}}-v_{\mathrm{GNSS}}$, are adopted in the process of measurement update. Therefore, the vector of the measurement $z_{k}$ is constructed below.

$$
z_{k}=\left[\begin{array}{c}
\Delta r \\
\Delta v
\end{array}\right],
$$

Actually, the measurement equation is linear, and the measurement updating process should be modified as follows.

$$
\begin{aligned}
x_{k / k} & =x_{k / k-1}+K_{k}\left(z_{k}-H_{k} x_{k / k-1}\right), \\
K_{k} & =P_{k / k-1} H_{k}^{T}\left(H_{k} P_{k / k-1} H_{k}^{T}+R_{k}\right)^{-1}, \\
P_{k / k} & =P_{k / k-1}-K_{k} H_{k} P_{k / k-1} .
\end{aligned}
$$

Obviously, the precision of the estimates relies largely on the quality of the measurements, and unreliable measurements may result in inestimable influences. In practice, however, unreliable measurements are inevitable. Therefore, the abnormal measurements must be deliberately processed. Both the fading factor or matrix and the covariance inflation factor have been adopted in the data processing of the GNSS/INS integrated systems. Aiming at the influences on the fading factor, the judging index constructed based on the Mahalanobis distance and the covariance inflation factor is implemented to improve the performance of the filtering process.

\section{Performance Evaluation and Analysis}

Data processing experiments using different filtering algorithms were designed and implemented. Equipped with the GNSS receiver (Trimble R8) and the inertial measurement unit (IMU, SPAN-CPT), the land vehicle was used as the testing carrier, and a three-axis open-loop gyroscope and three-axis MEMS accelerometers are included in the IMU. 


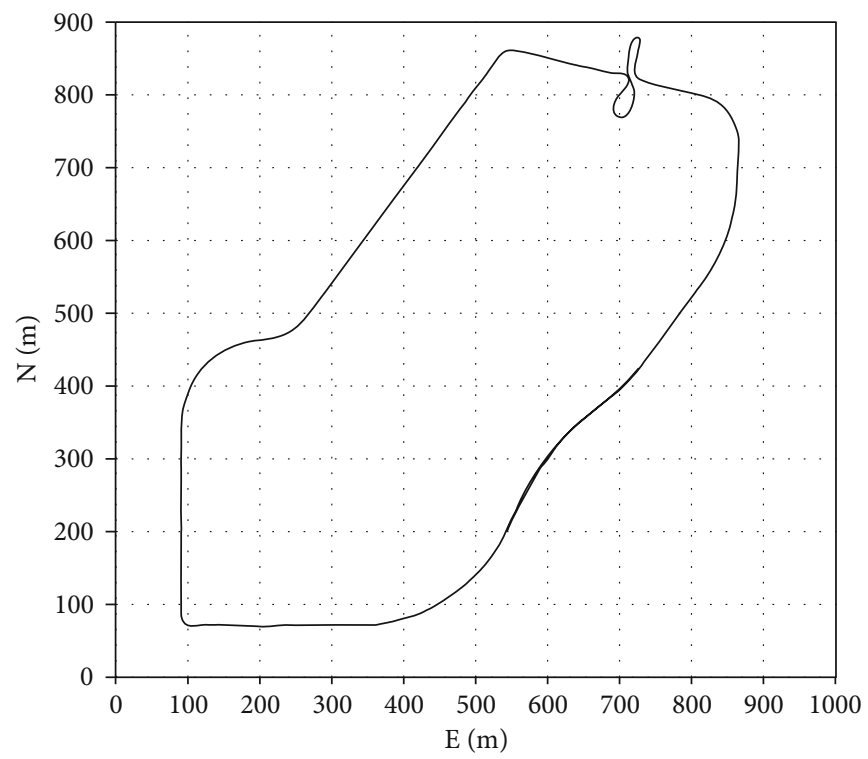

FIgURE 1: Motion trajectory of the land vehicle.

TABle 1: Main technical parameters from the suppliers.

\begin{tabular}{lccccc}
\hline Sensors & Constant bias & Bias instability & Bias repeatability & Random walk & Scale factor instability \\
\hline Gyroscope & $20^{\circ} / \mathrm{h}$ & $1^{\circ} / \mathrm{h}(1 \sigma)$ & $3^{\circ} / \mathrm{h}$ & $0.0667^{\circ} / \mathrm{h}^{1 / 2}$ & $1500 \mathrm{ppm}$ \\
Accelerometer & $50 \mathrm{mg}$ & $0.25 \mathrm{mg}(1 \sigma)$ & $0.75 \mathrm{mg}$ & $50 \mu \mathrm{g} / \mathrm{h}^{1 / 2}$ & $4000 \mathrm{ppm}$ \\
\hline
\end{tabular}

The average speed of the land vehicle was about $3.5 \mathrm{~m} / \mathrm{s}$. Meanwhile, another GNSS receiver called the base station was set on top of the building. Motion trajectory of the land vehicle (in the North and East directions) is demonstrated in Figure 1. The main technical parameters provided by the suppliers were listed in Table 1. Then, the data was collected with the land vehicle under real conditions. Position and velocity from GNSS were computed using double-difference pseudorange measurement, and the position and velocity deviations were $0.25 \mathrm{~m}^{2}$ and $(0.05 \mathrm{~m} / \mathrm{s})^{2}$, respectively. In the testing systems, the cut-off angle was set by $10^{\circ}$, and the sampling frequency was $1 \mathrm{~Hz}$ for GNSS and $100 \mathrm{~Hz}$ for INS. In this paper, the tightly coupled results provided by the commercial software IE using double-difference carrier phase measurements were regarded as the references.

As to data fusion of the GNSS/INS integrated systems of the paper, the CKF was adopted as the basic filter, and time interval of the data fusion was one second. In the experiments, both the initial data and the data with artificially added outliers were adopted to test the performances of different algorithms more clearly. Consequently, two cases were designed and performed. Each case consists of four algorithms, and the differences between the references and the results of each algorithm are thought to be errors. Comparing the fading filters with a fading factor and fading matrix, the fading matrix performs better by adjusting the covariance matrix in multiple data channels simultaneously, and the ability to resist the model mismatch is enhanced. Therefore, the fading matrix was adopted in this paper. For all algorithms of this paper, the initial value of the covari- ance are fixed by experience. Thus, the statistical deviations of the models exist, and it is suitable to test the proposed estimation algorithm. Four different algorithms of each case are designed below.

Algorithm 1. The conventional CKF.

Algorithm 2. The fading filter with fading matrix (MF-CKF).

Algorithm 3. The fading filter with fading matrix is implemented at the epochs when the null hypothesis is accepted (Partial-MF-CKF).

Algorithm 4. The fading filter with fading matrix is implemented while the null hypothesis is accepted; otherwise, the covariance inflation factor is adopted for outlying measurements and sequential updating for normal measurements (RMF-CKF).

Case 5. In this case, the initial data collected through the selfdeveloped GNSS/INS integrated navigation systems is processed with the above four algorithms, respectively. Positioning errors of the land vehicle of each algorithm are demonstrated in Figures 2-5.

As mentioned above, each algorithm was implemented based on the initial measurements collected under ideal observation conditions, and little outliers exist in the measurements. Accordingly, positioning errors were mainly brought by the model errors. Comparing Figures 2-5, apparently, error amplitudes in $X, Y$, and $Z$ directions of the CKF 

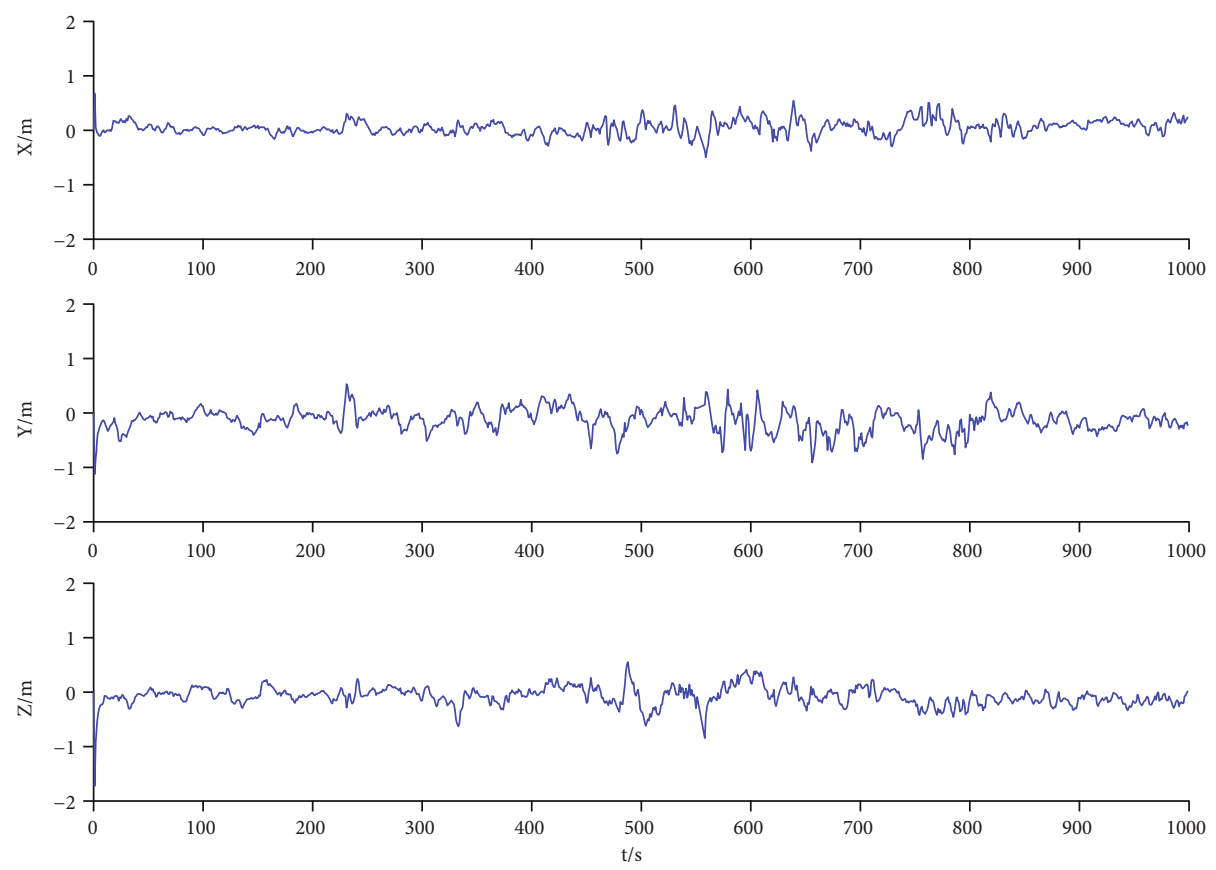

FIgURe 2: Position errors of the CKF algorithm.
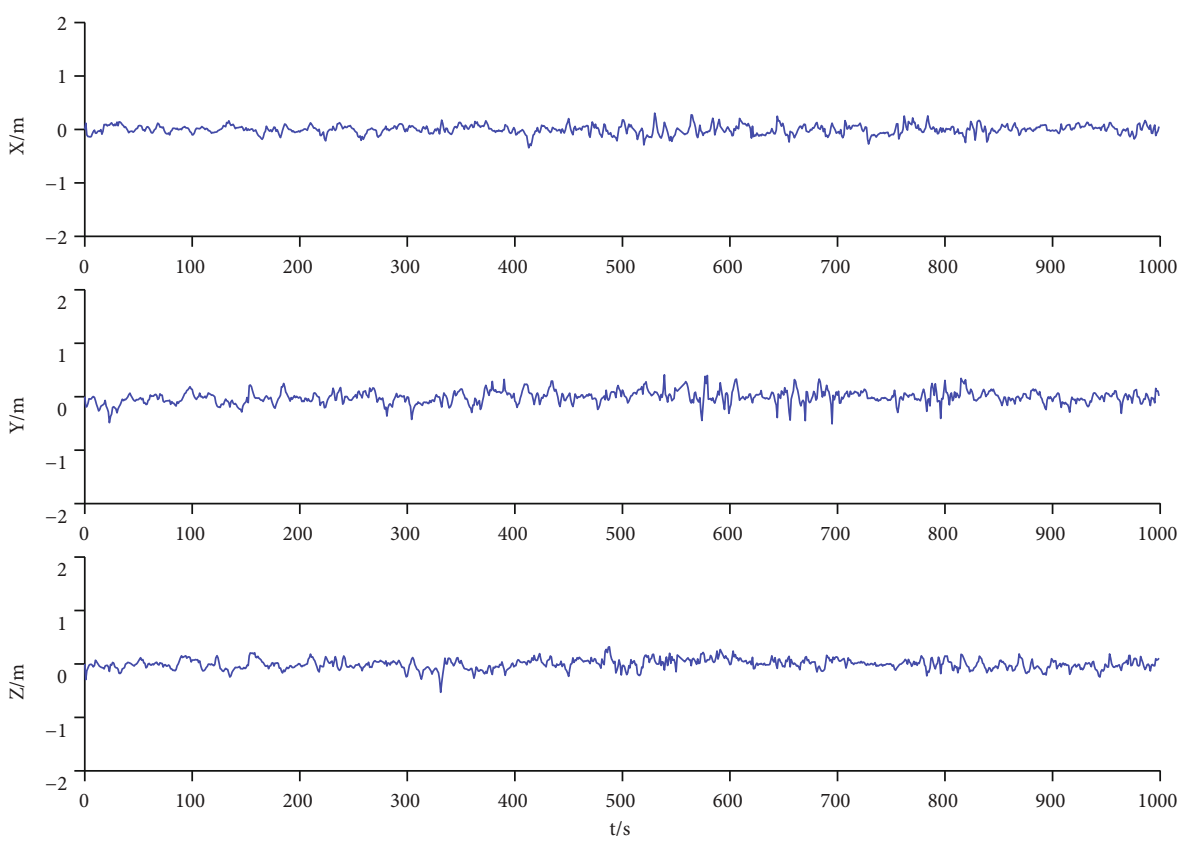

Figure 3: Position errors of the MF algorithm.

algorithm are much bigger than the other algorithms, and this indicates that the performance of the conventional CKF algorithm is improved significantly. Moreover, stability of the filter was improved, and the filter divergence resulted from the model uncertainties was restrained effectively with the last three algorithms. In theory, main differences between the Algorithms 2 and 3 lie on the circumstance when the fading matrix is adopted. With the initial measurements, the null hypothesis was accepted at most epochs; thus, the results of the Algorithms 2 and 3 are similar. In the MF-CKF algorithm, the fading matrix was implemented at all epochs, and the model errors were well-considered. Except for the epochs when the null hypothesis was accepted, the covariance inflation factor in the RMF algorithm was adopted at other epochs, and the influences of abnormal measurements were weakened. Consequently, error amplitudes of the processed algorithm displayed in Figure 4 are smaller than those of other three algorithms.

The root mean square error (RMSE) of these positioning errors was computed to examine the performances of each 

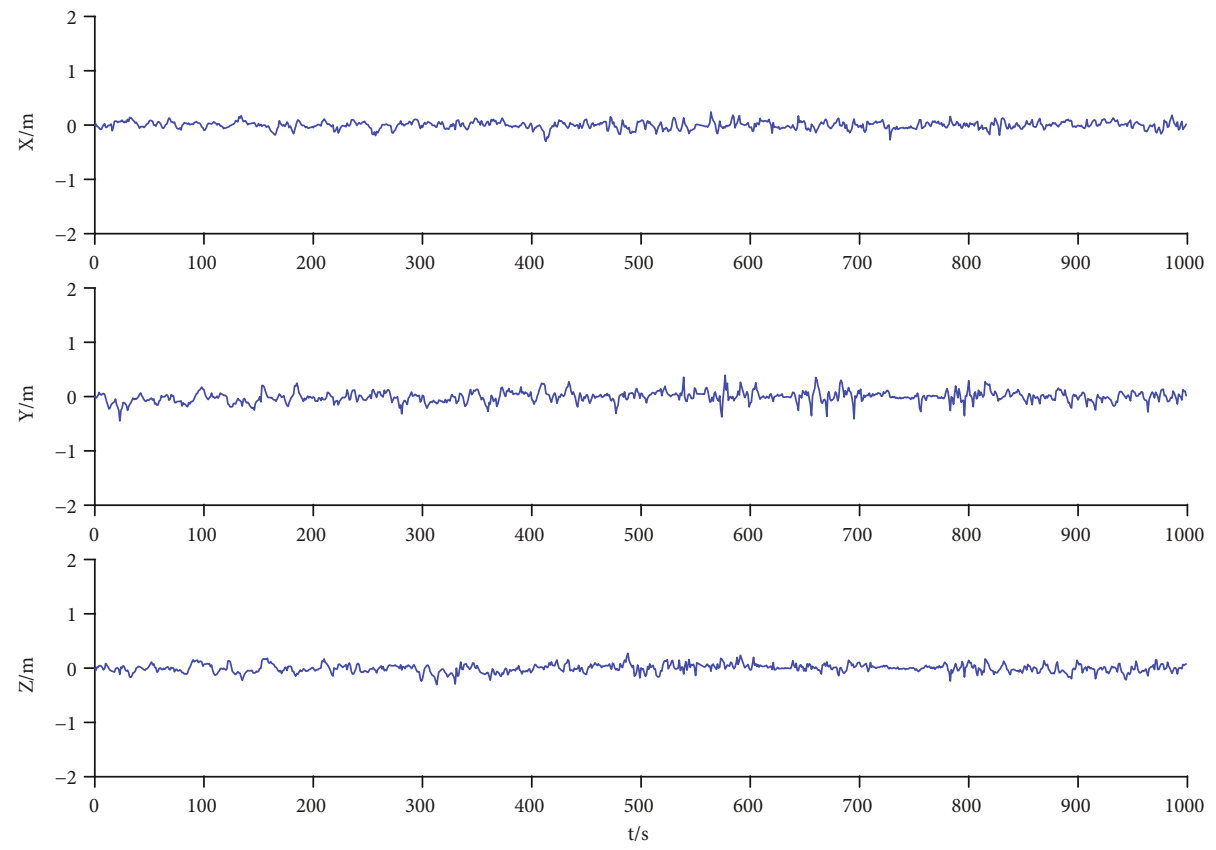

FIgure 4: Position errors of the Partial-MF-CKF algorithm.
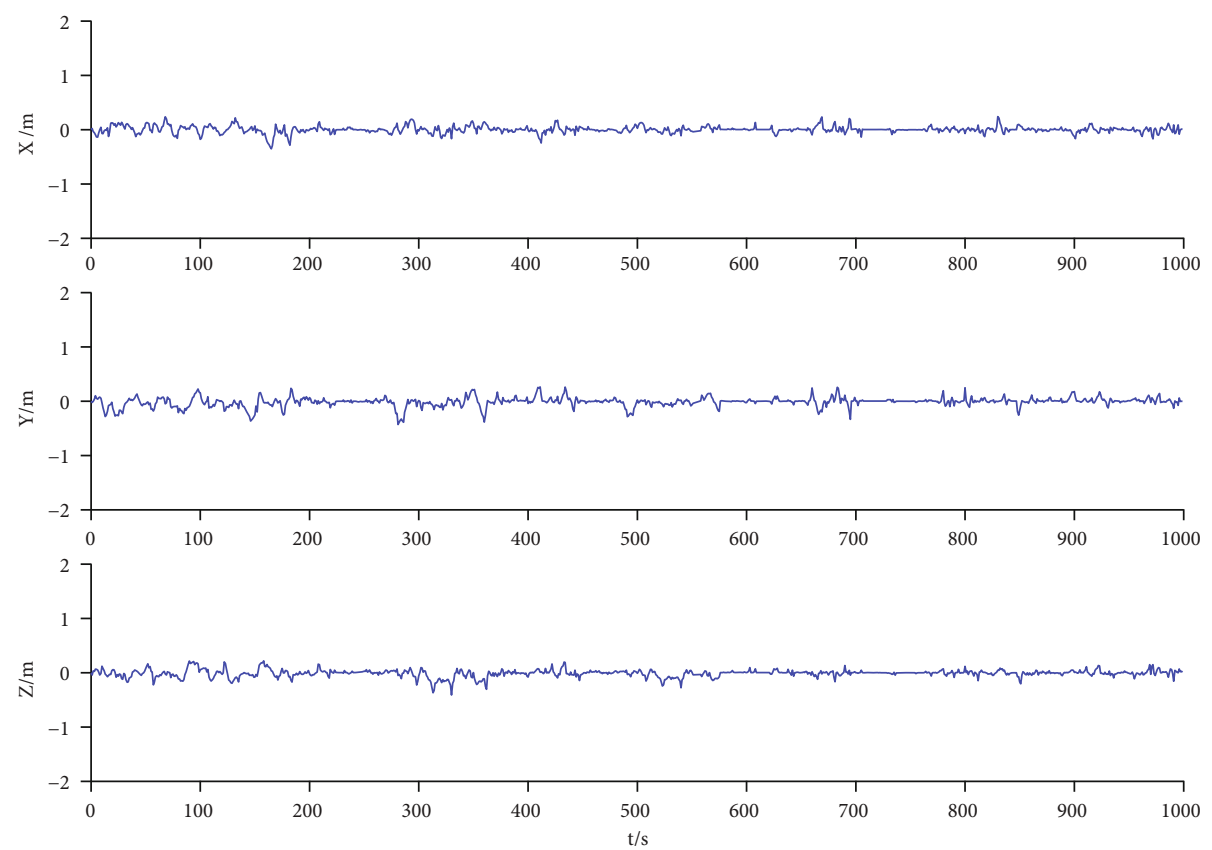

Figure 5: Position errors of the RMF-CKF algorithm.

algorithm quantificationally. Figure 6 displays RMSEs in $X$ ,$Y$, and $Z$ directions of each algorithm. Meanwhile, the detailed RMSEs (position $\left(P_{X}, P_{Y}\right)$, velocity $\left(V_{X}, V_{Y}\right)$, and attitude (Yaw) are taken as examples) of these four algorithms are listed in Table 2.

In terms of RMSE values in Figure 6 and Table 2, CKF is much bigger than other three algorithms, and this indicates that the robustness and stability of the conventional CKF should be enhanced further. Comparing the RMSE values of MF-CKF and Partial-MF-CKF algorithms, the former performed better since the influences of model errors were more significant than those of abnormal measurements. Based on the Partial-MF-CKF, the covariance inflation factor was implemented at the epochs when the null hypothesis was rejected. Thus, superiorities of the Mahalanobis distance, the fading filter, and the robust estimation are combined in the proposed algorithm. Meanwhile, RMSE values of the other schemes are all bigger than those of the RMF- 


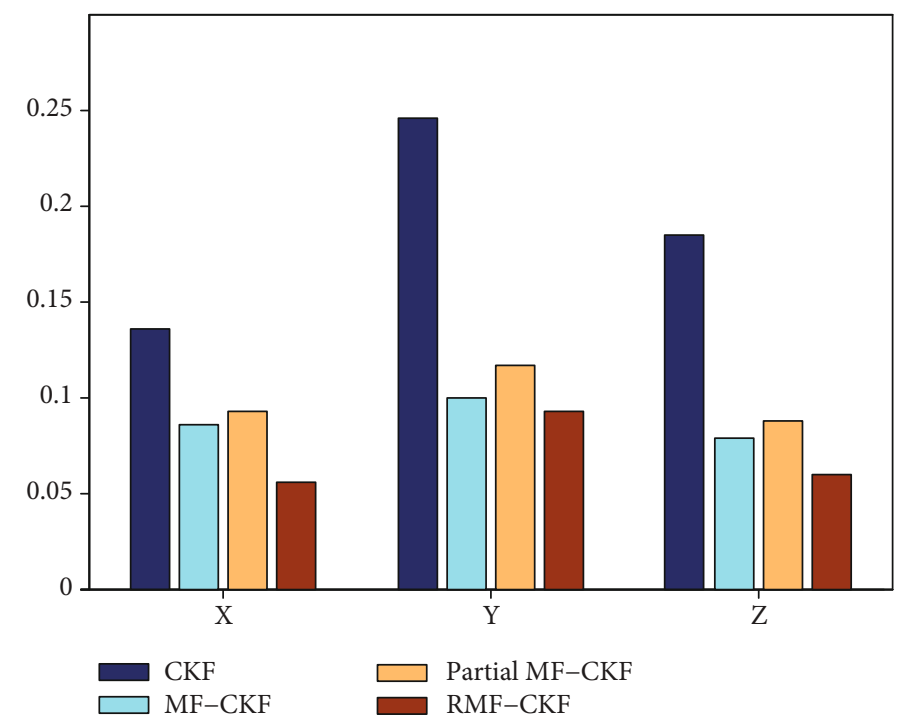

FIGURE 6: Positioning error RMSEs of different algorithms (m). In $X, Y$, and $Z$ directions, RMSEs of the Algorithms 1, 2, 3, and 4 are depicted in order from the left to the right, respectively.

TABLE 2: Error RMSEs of different algorithms.

\begin{tabular}{lccccc}
\hline Algorithm & $P_{X}(\mathrm{~m})$ & $P_{Y}(\mathrm{~m})$ & $V_{X}(\mathrm{~m} / \mathrm{s})$ & $V_{Y}(\mathrm{~m} / \mathrm{s})$ & Yaw $\left(^{\circ}\right)$ \\
\hline CKF & 0.147 & 0.270 & 0.186 & 0.160 & 6.242 \\
MF-CKF & 0.079 & 0.100 & 0.099 & 0.096 & 4.169 \\
Partial-MF-CKF & 0.093 & 0.117 & 0.104 & 0.098 & 4.318 \\
RMF-CKF & 0.065 & 0.087 & 0.056 & 0.061 & 3.620 \\
\hline
\end{tabular}

CKF algorithm, and this indicates that better performance is achieved with the processed algorithm. In Table 2, obviously, RMSEs of all the fading filtering algorithms are smaller than the CKF algorithm since effects of the model errors are weakened, and the RMF-CKF algorithm outperforms the other algorithms in terms of accuracy. Compared with the conventional MF algorithm, the accuracy of positioning is improved for $17.7 \%$ and $13.0 \%$ in $X$ and $Y$ directions, respectively, using the RMF-CKF algorithm.

Case 6. In this case, the continuously changing and the separated positioning outliers were introduced artificially into the measurements to test the robustness and stability of each algorithm. Thus, the perturbation data based on the initial data was constructed. All the above four algorithms were implemented based on the perturbation data, and positioning errors of each algorithm are illustrated in Figures 7-10.

Since positioning outliers were introduced into the measurements, the outliers, instead of the model errors, became the principal factor in affecting the filtering performance. It is demonstrated in Figures 7-10 that amplitudes of all algorithms were much bigger than those of Case 1, and this denotes that all algorithms were affected significantly by the outlying measurements. Meanwhile, as to error amplitudes, all the algorithms with fading matrix are better than the CKF algorithm. Since the outlying measurements were not addressed effectively in the CKF and Partial-MF-CKF algorithms, Figures 7 and 9 illustrate that effects of the outlying measurements are more apparent. As shown in Figures 7 and 9, the fading matrix was implemented when the null hypothesis was accepted and the model errors were well controlled; thus, the Partial-MF-CKF algorithm performed superior than the CKF. In the MF-CKF algorithm, the fading matrix is implemented at all epochs; the fading matrix performs better when the null hypothesis is accepted. However, the fading filter becomes instable when the null hypothesis is rejected. Therefore, once the fading matrix is applied mistakenly, the MF-CKF algorithm may become inferior to the CKF algorithm. Comparing Figures 7 and 8, it is illustrated that amplitudes at some epochs of the MFCKF algorithm are even bigger than those of the CKF algorithm, and this is the influences brought from the outlying measurements. In other words, implementing the fading matrix mistakenly may affect the filtering performance at the current together with the future epochs. In the RMFCKF algorithm, both the model errors and the outlying measurements were considered. Meanwhile, the fading matrix was implemented at the relatively reasonable epochs. In Figure 10, amplitudes in three directions are much smaller than the other algorithms, and a better performance is achieved with the RMF-CKF algorithm. However, as to the constantly changing outliers, the values may small enough to go through the hypothesis test, and the fading matrix will be implemented mistakenly. Therefore, at some epochs in Figure 10, performance of the RMF-CKF algorithm was still apparently affected by the constantly changing outliers.

Figure 11 depicts RMSEs of each algorithm, and the detailed RMSEs of these four algorithms are provided in Table 3.

Comparing the RMSEs in Figures 6 and 11, it is concluded that all algorithms were affected by the outlying measurements. Under the effects of outlying measurements, the model errors were weakened effectively; thus, the MF-CKF algorithm performed better than the CKF algorithm. 

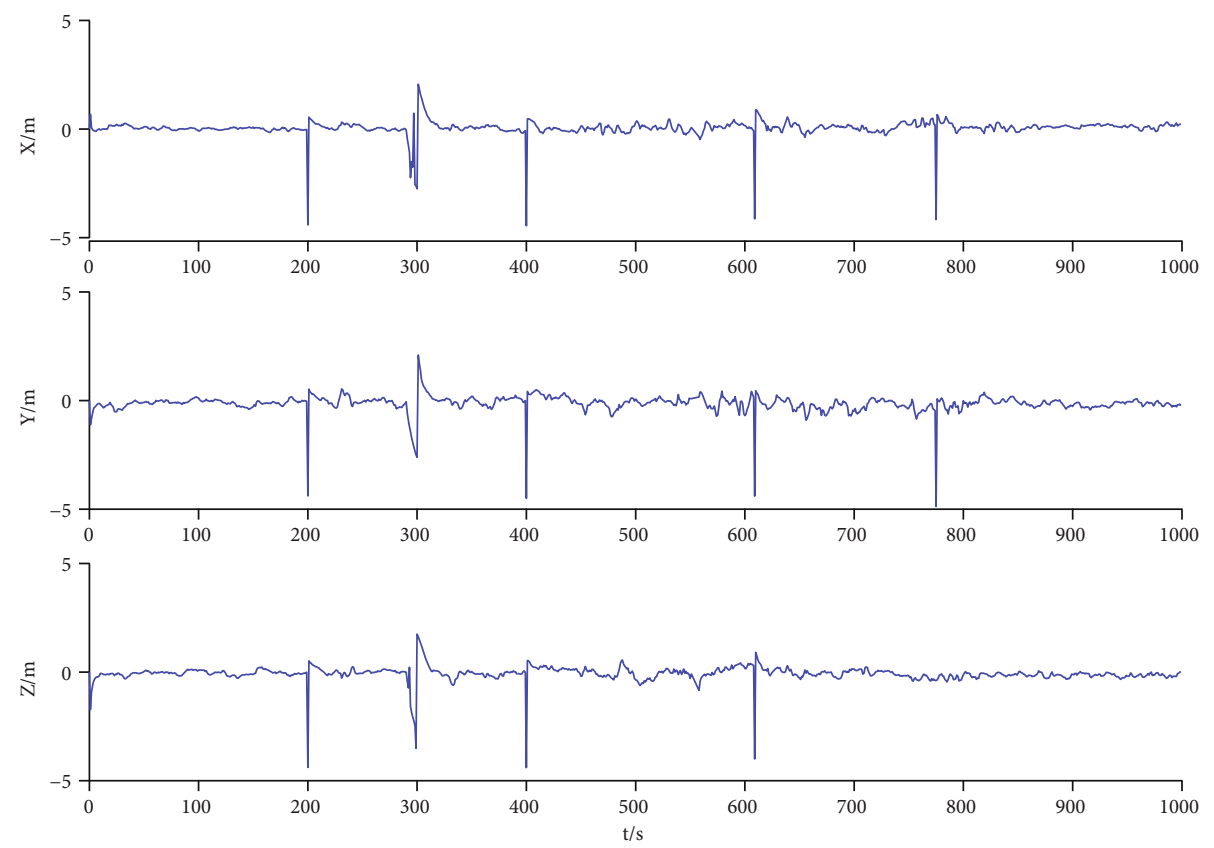

FIgUre 7: Position errors of the CKF algorithm.
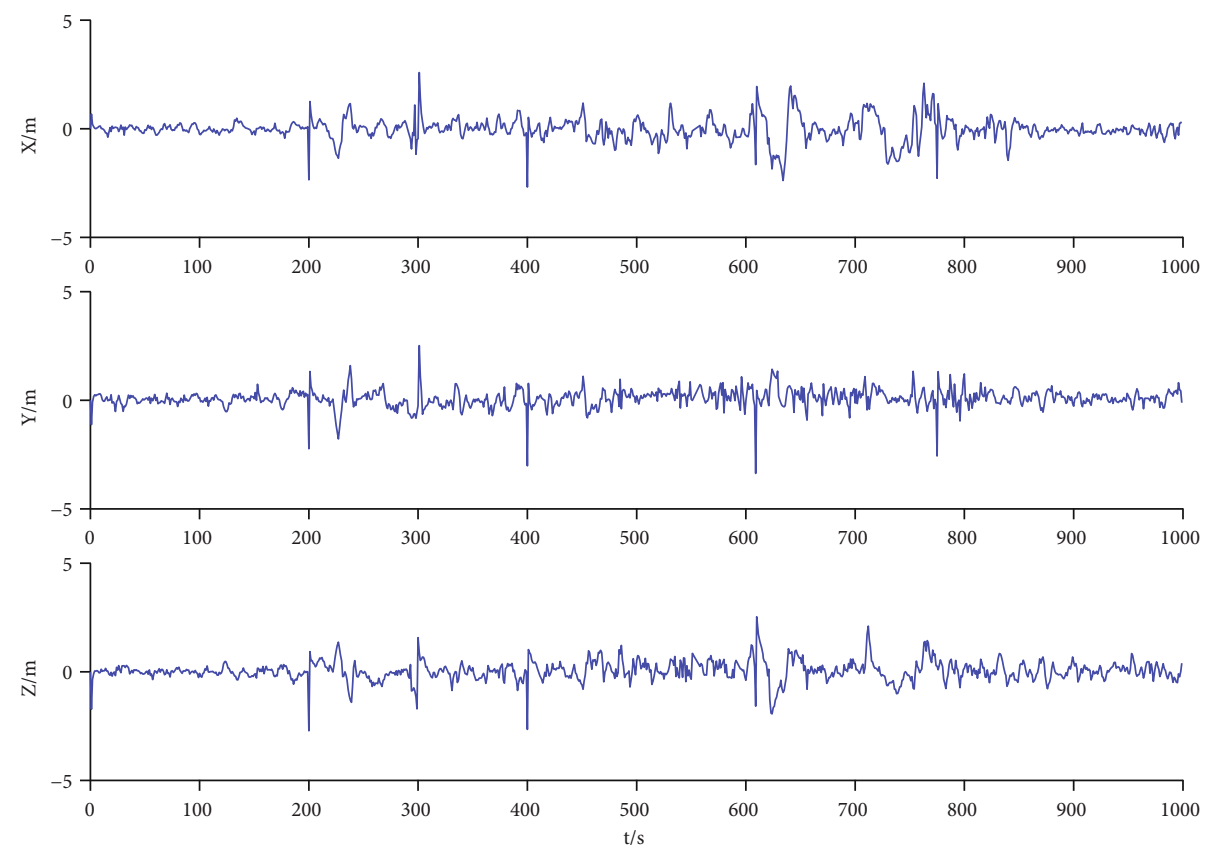

Figure 8: Position errors of the MF-CKF algorithm.

Although both the MF and Partial-MF algorithms were affected significantly by the outlying measurements, the fading matrix was implemented only when the null hypothesis was accepted, and the negative effects from mistakenly used fading matrix were avoided in the Partial-MF-CKF algorithm. Therefore, the Partial-MF-CKF algorithm performed better than the MF-CKF algorithm. Corresponding to the qualitative conclusions, RMSEs of the RMF-CKF algorithm are much smaller than the other algorithms; apparently, better performance is achieved with the proposed algorithm.
Comparing Tables 2 and 3, it is demonstrated that the performances of all algorithms were degraded by the outlying measurements, and the filters with fading matrix are more stable and robust than CKF algorithm. By integrating the superiorities of the multiple fading filter and the robust estimation method, the smaller RMSE values and a relatively stable performance were obtained with the proposed algorithm. In this circumstance, the accuracy of positioning are improved for $60.1 \%$ and $58.5 \%$ in $X$ and $Y$ directions, respectively, using the $\mathrm{RMF}-\mathrm{CKF}$ algorithm, and this in turn 

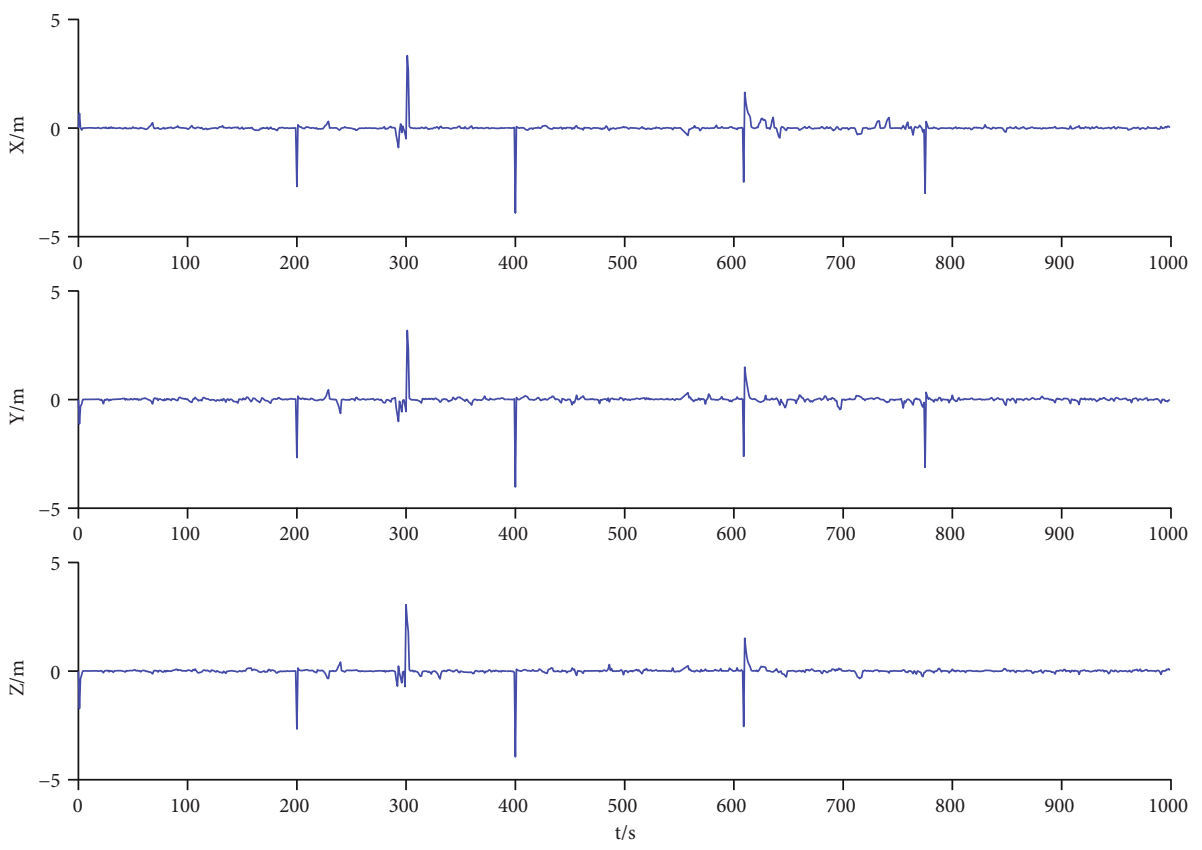

FIgure 9: Position errors of the Partial-MF-CKF algorithm.
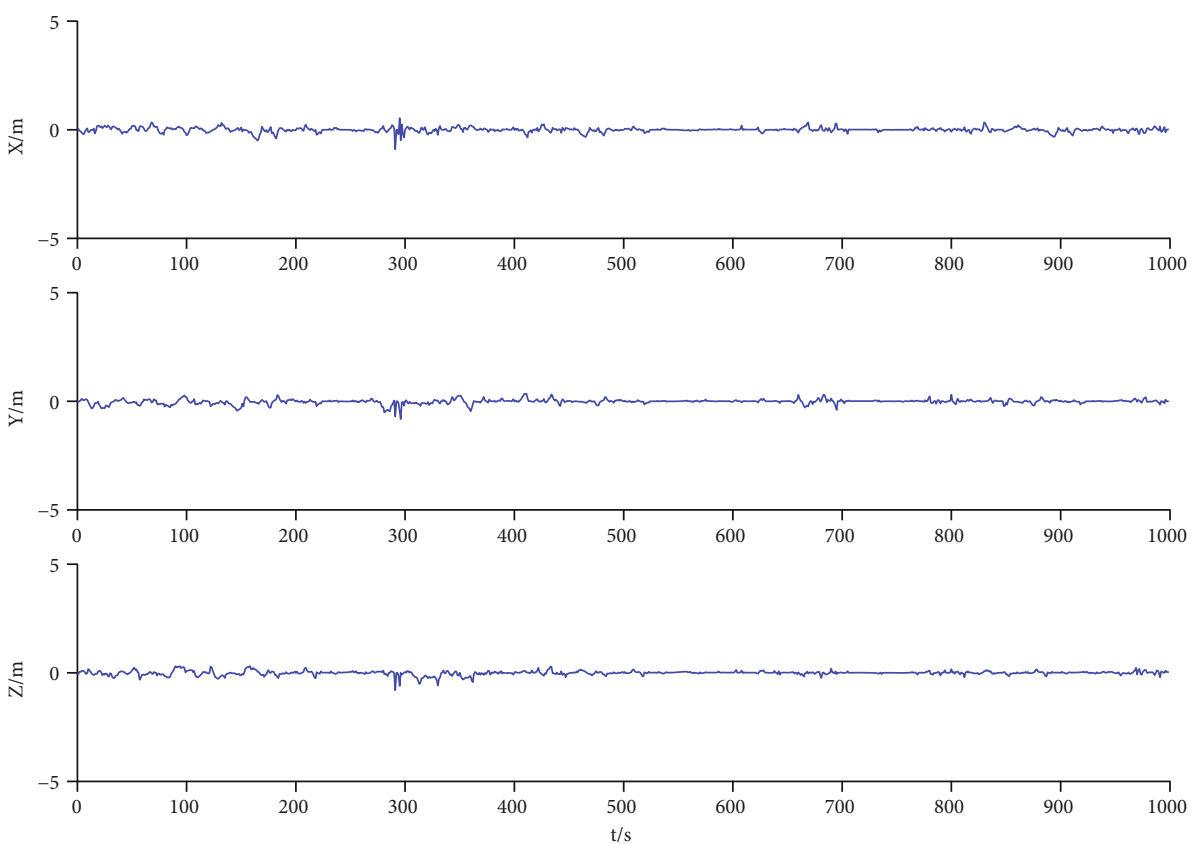

FIgURE 10: Position errors of the RMF-CKF algorithm.

demonstrates that the MF algorithm is affected by the outlying measurements seriously.

\section{Conclusions}

In this paper, a rarely discussed problem about how to apply the fading factor or matrix with unreliable measurements adaptively is researched, and a modified adaptive filter and an alternative scheme are developed. In the proposed scheme, the hypothesis test was performed using the statistics derived from the Mahalanobis distance, and the actual measurements were collected with self-developed GNSS/ INS integrated systems; then, the contrastive experiments and analysis were implemented to test the performance of the proposed algorithm. Detailed conclusions of this paper are summarized below:

(1) Compared with the conventional CKF, the adaptive filter with the fading matrix can control the negative effects of model uncertainties; however, the performance may be degraded significantly when there exist outlying measurements 


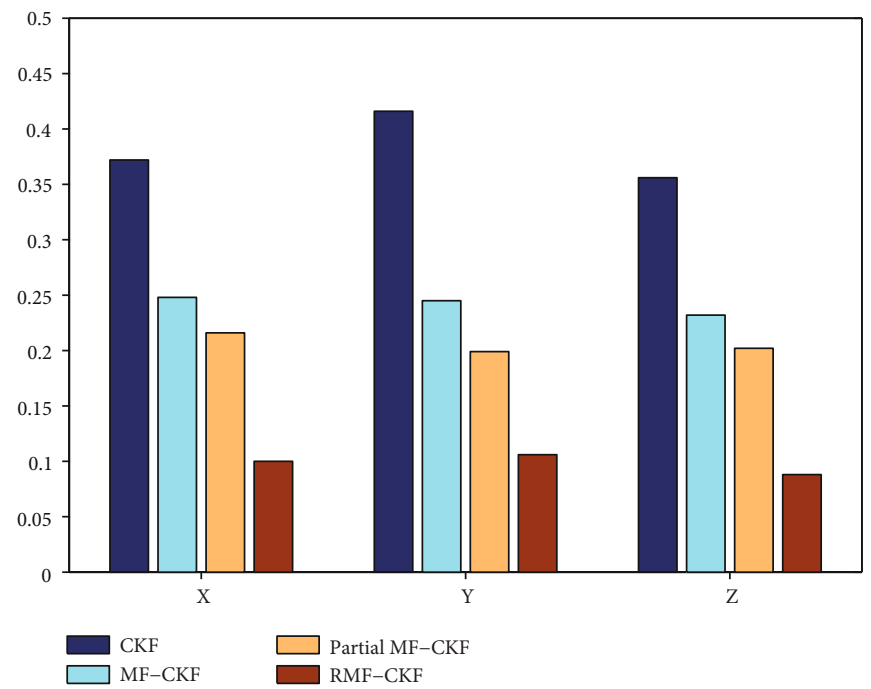

Figure 11: Error RMSE of different algorithms (m). In $X, Y$, and $Z$ directions, RMSEs of the Algorithms 1, 2, 3, and 4 are depicted in order from the left to the right, respectively.

TABLE 3: Error RMSEs of different algorithms.

\begin{tabular}{lccccc}
\hline Algorithm & $P_{X}(\mathrm{~m})$ & $P_{Y}(\mathrm{~m})$ & $V_{X}(\mathrm{~m} / \mathrm{s})$ & $V_{Y}(\mathrm{~m} / \mathrm{s})$ & Yaw $\left(^{\circ}\right)$ \\
\hline CKF & 0.392 & 0.436 & 0.189 & 0.162 & 6.403 \\
MF-CKF & 0.263 & 0.265 & 0.120 & 0.104 & 5.615 \\
Partial-MF-CKF & 0.231 & 0.209 & 0.108 & 0.095 & 5.127 \\
RMF-CKF & 0.105 & 0.110 & 0.069 & 0.078 & 4.351 \\
\hline
\end{tabular}

(2) In the fading filter, unreliable measurements will construct unreliable fading matrix, and performance of the filter may be even inferior to the conventional Kalman filter if the fading matrix is applied mistakenly. Therefore, it is suggested that the fading matrix be applied in a timely and more reasonable way

(3) Statistics derived from the Mahalanobis distance can be adopted to test the quality of measurements, and stability of the fading filter is improved with the hypothesis test. By integrating the advantages of the fading matrix and the covariance inflation factor, effects of both the model errors and the outlying measurements are controlled using the proposed scheme for the systems with model uncertainties. Also, the proposed adaptive fusion scheme is more suitable when there exist outliers in the measurements

\section{Data Availability}

Some or all data, models, or code that support the findings of this study are available from the corresponding author upon reasonable request (email: xuhaijun@ncwu.edu.cn).

\section{Conflicts of Interest}

The authors declare no conflict of interest.

\section{Acknowledgments}

This research project was supported by the Natural Science Foundation of Henan Province (212300410198), the Nation Key Research Program of China (2017YFE0131400), and the State Key Laboratory of Geodesy and Earth's Dynamics (SKLGED 2020-3-1-E).

\section{References}

[1] G. B. Chang, "Robust Kalman filtering based on Mahalanobis distance as outlier judging criterion," Journal of Geodesy, vol. 88, no. 4, pp. 391-401, 2014.

[2] R. E. Kalman, "A new approach to linear filtering and prediction problems," Journal of Basic Engineering, vol. 82, no. 1, pp. 35-45, 1960.

[3] M. Aslinezhad, A. Malekijavan, and P. Abbasi, "ANN-assisted robust GPS/INS information fusion to bridge GPS outage," EURASIP Journal on Wireless Communications and Networking, vol. 1, 18 pages, 2020.

[4] F. A. L. Costa and E. A. Mitishita, "An approach to improve direct sensor orientation using the integration of photogrammetric and lidar datasets," International Journal of Remote Sensing, vol. 40, no. 14, pp. 5651-5672, 2019.

[5] D. Simon, Optimal State Estimation: Kalman, H Infinity, and Nonlinear Approaches, John Wiley \& Sons, 2006.

[6] X. Xu, Y. Sun, Y. Yao, and T. Zhang, "A robust in-motion optimization-based alignment for SINS/GPS integration," IEEE Transactions on Intelligent Transportation Systems, vol. 99, pp. 1-11, 2021.

[7] R. Ihsan, S. Almufti, and R. Marqas, "A median filter with evaluating of temporal ultrasound image for impulse noise removal for kidney diagnosis," Journal of Applied Science and Technology Trends, vol. 1, no. 2, pp. 71-77, 2020.

[8] G. Rigatos, P. Siano, P. Wira, K. Busawon, and R. Binns, “A nonlinear $\mathrm{H}$-infinity control approach for autonomous truck and trailer systems," Unmanned Systems, vol. 8, no. 1, pp. 49-69, 2020. 
[9] Y. X. Yang, "Robust Bayesian estimation," Journal of Geodesy, vol. 65, no. 3, pp. 145-150, 1991.

[10] Y. X. Yang, H. B. He, and G. Xu, "Adaptively robust filtering for kinematic geodetic positioning," Journal of Geodesy, vol. 75, no. 2-3, pp. 109-116, 2001.

[11] A. H. Mohamed and K. P. Schwarz, "Adaptive Kalman filtering for INS/GPS,” Journal of Geodesy, vol. 73, no. 4, pp. 193203, 1999.

[12] C. Sadhukhan, S. Mitra, M. Naskar, and M. Sharifpur, "Fault diagnosis of a nonlinear hybrid system using adaptive unscented Kalman filter bank," Engineering with Computers, vol. 2, pp. 1-12, 2021.

[13] R. P. Tripathi, A. K. Singh, and P. Gangwar, "Innovation-based fractional order adaptive Kalman filter," Journal of Electrical Engineering, vol. 71, no. 1, pp. 60-64, 2020.

[14] R. Mehra, "On the identification of variances and adaptive Kalman filtering," IEEE Transactions on Automatic Control, vol. 15, no. 2, pp. 175-184, 1970.

[15] Y. X. Yang, X. Ren, and Y. Xu, "Main progress of adaptively robust filter with applications in navigation," Journal of Navigation and Positioning, vol. 1, no. 1, pp. 9-15, 2013.

[16] C. Jiang and S. B. Zhang, "A novel adaptively-robust strategy based on the Mahalanobis distance for GPS/INS integrated navigation systems," Sensors, vol. 18, no. 3, p. 695, 2018.

[17] E. Zerdali, R. Yildiz, R. Inan, R. Demir, and M. Barut, "Improved speed and load torque estimations with adaptive fading extended Kalman filter," International Transactions on Electrical Energy Systems, vol. 31, no. 10, p. 12684, 2021.

[18] Y. R. Geng and J. Wang, "Adaptive estimation of multiple fading factors in Kalman filter for navigation applications," GPS Solutions, vol. 12, no. 4, pp. 273-279, 2008.

[19] H. M. Qian, G. E. Lei, and P. Yu, "Multiple fading factors Kalman filter and its application in SINS initial alignment," Journal of Chinese Inertial Technology, vol. 20, pp. 287-291, 2012.

[20] Y. R. Chen and J. P. Yuan, "An improved robust Ho multiple fading fault-tolerant filtering algorithm for INS/GPS integrated navigation," Journal of Astronautics, vol. 30, no. 3, pp. 930-936, 2009.

[21] Y. X. Yang, Adaptive Navigation and Kinematic Positioning (the Second Version), Surveying and Mapping Press, Beijing, China, 2017.

[22] Q. J. Xia, Y. X. Sun, and C. H. Zhou, "An optimal adaptive algorithm for fading Kalman filter and its application," Acta Automatica Sinica, vol. 16, no. 3, pp. 210-216, 1990.

[23] A. J. Izenman, Modern Multivariate Statistical Techniques: Regression, Classification, and Manifold Learning, Springer, Berlin, 2008.

[24] Y. Wang, J. Liu, J. Wang, Q. Zeng, X. Shen, and Y. Zhang, "Micro aerial vehicle navigation with visual-inertial integration aided by structured light," The Journal of Navigation, vol. 73, no. 1, pp. 16-36, 2020.

[25] K. W. Chiang, G. J. Tsai, H. W. Chang, C. Joly, and N. EISheimy, "Seamless navigation and mapping using an INS/ GNSS/grid-based SLAM semi-tightly coupled integration scheme," Information Fusion, vol. 50, pp. 181-196, 2019.

[26] S. Hasheminasab, T. Zhou, and A. Habib, "GNSS/INS-assisted structure from motion strategies for UAV-based imagery over mechanized agricultural fields," Remote Sensing, vol. 12, no. 3, p. 351, 2020.
[27] I. Arasaratnam and S. Haykin, "Cubature Kalman filters," IEEE Transactions on Automatic Control, vol. 54, no. 6, pp. 1254-1269, 2009.

[28] G. Reina, A. Leanza, and A. Messina, “Terrain estimation via vehicle vibration measurement and cubature Kalman filtering," Journal of Vibration and Control, vol. 26, no. 11-12, pp. 885-898, 2020. 